\title{
A Neuro-Fuzzy Hybridization Approach to Model Weather Operations in a Virtual Warfare Analysis System
}

\author{
D. Vijay Rao ${ }^{1}$, Lazaros Iliadis ${ }^{2}$, and Stefanos Spartalis ${ }^{3}$ \\ ${ }^{1}$ Institute for Systems Studies and Analyses \\ Defence Research and Development Organisation, \\ Metcalfe House, Delhi 110054, India \\ \{doctor.rao.cs@gmail.com\} \\ ${ }^{2}$ Department of Forestry \& Management of the Environment and Natural Resources \\ Democritus University of Thrace, GR-68200, Orestiada, Hellas \\ liliadis@fmenr.duth.gr \\ ${ }^{3}$ Department of Production Engineering and Management \\ Democritus University of Thrace, GR-68200, Orestiada, Hellas \\ sspart@pme.duth.gr
}

\begin{abstract}
Weather operations play an important and integral part of planning, execution and sustainment of mission operations. In this paper, a neuro-fuzzy hybridization technique is applied to model the weather operations and predict its impact on the effectiveness of air tasking operations and missions. Spatiotemporal weather data from various meteorological sources are collected and used as the input to a neural network and the predicted weather conditions at a given place is classified based on fuzzy logic. The corresponding fuzzy rules are generated forming the basis for introducing the weather conditions in the evaluation of the effectiveness of the military mission plans. An agent-based architecture is proposed where agents representing the various weather sensors feed the weather data to the simulator, and a weather agent developed using neuro-fuzzy hybridization computes the weather conditions over the flight plan of the mission. These rules are then used by the Mission Planning and Execution system that evaluates the effectiveness of military missions in various weather conditions.
\end{abstract}

Keywords: Military simulation, Weather Modeling, Neuro-Fuzzy hybridization, Mission effectiveness, agent-based architecture.

\section{Introduction}

Virtual Warfare Analysis systems constitute an important class of applications that have proved to be an important tool for military system analyses and an inexpensive alternate to live training exercises. Air Wargame Simulation System (AWGSS) is a virtual warfare analysis software that has been developed for planning, analysis and evaluating air tasking operations [1],[2]. In the design and development of such applications, modeling the complexity and battle dynamics, assessing, and predicting 
the outcomes of mission plans quantitatively under various real-world conditions is a very difficult endeavor [3],[4]. One such phenomenon that bears an important and critical success factor in achieving the military objectives is weather operations [1]. Weather operations [5] are an integral part of planning, execution, and sustainment of military operations and critical to commanders' battle space awareness and decision making across the range of military operations. Operational environment and weather conditions play an important role in deciding the suitability of the weapon for a target. For instance, poor weather and conflict induced environmental conditions such as smoke from bombing may degrade or block the infrared, electro-optical or laser targeting sensors required for delivery of guidance ordnance. Laser Guided Bombs cannot be used in poor weather where target illumination cannot be seen, or where it is not possible to get target designator in close distance. However, poor visibility does not affect satellite guided bombs. Only comparatively inaccurately unguided bombs could be delivered in poor weather. These factors change the decisions taken by the pilots on Execute/Call-off mission, Change mission route and/or profile of weapon delivery, change in the class of weapons to deliver in the light of sensors performance, re-prioritize the target, and/or change the Time-on-target of the mission or even abort the mission [1],[2]. In this paper, ANFIS a neuro-fuzzy hybridization technique is used to model the weather conditions and predict the Mission_Effectiveness_Factor.

\section{An Agent-Based Architecture to Model Military Operations}

Agent-oriented system development aims to simplify the construction of complex systems by introducing a natural abstraction layer on top of the object-oriented paradigm composed of autonomous interacting actors. It has emerged as a powerful modeling technique that is more realistic for today's dynamic warfare scenarios than the traditional models which were deterministic, stochastic or based on differential equations. These approaches provide a very simple and intuitive framework for modeling warfare and are very limited when it comes to representing the complex interactions of real-world combat because of their high degree of aggregation, multiresolution modeling and varying attrition rate factors. The effects of random individual agent behavior and of the resulting interactions of agents are phenomenon that traditional equation-based models simply cannot capture. The traditional approaches of computers to warfare simulation used algorithms that aggregated the forces on each side, such as differential equations or game theory, effectively modeling the entire battle space with a single process. These mathematical theories treat the opposing sides as aggregates, and do not consider the detailed interactions of individual entities.

Agent-based models give each entity its own thread of execution, mimicking the real-world entities that affect military operations [3]. The Environment Agent is a lightweight agent that determines the current environmental conditions over the area of operation selected for the mission. 


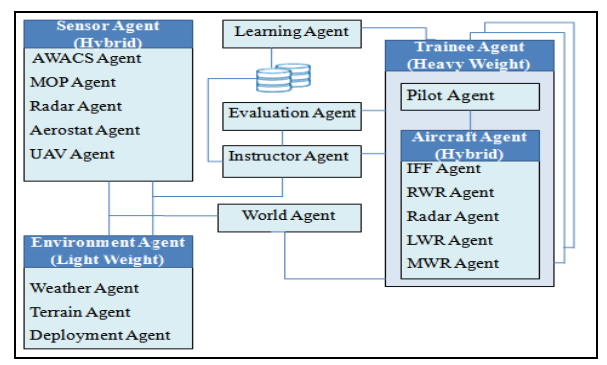

Fig. 1. Agent architecture for AWGSS

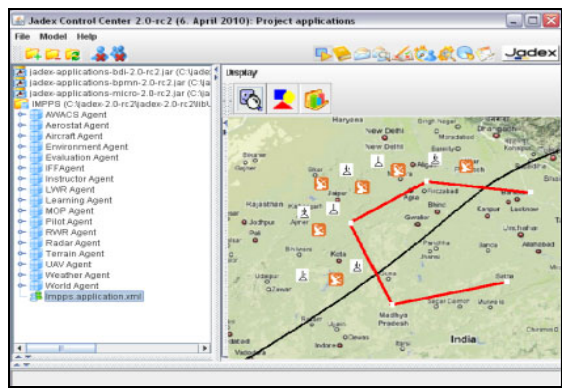

Fig. 2. Mission Planning with weather constraints

It receives information from weather, terrain and deployment agent and provides an information service to the world agent after its own process of reasoning. This information is then used by other agents such as Manual Observation Post (MOP), Pilot, Unmanned Air Vehicle (UAV), Identification Friend/Foe (IFF), Radar Warning Receiver (RWR), Missile Warning Receiver (MWR), Laser Warning Receiver (LWR), Mission Planning, Sensor Performance, Target Acquisition and Damage Assessment and Computation. The weather agent is an important agent that thas functions such as Get_Visibility(), Get_Temperature() and Get CloudCover(). The weather agents's reasoning has been designed using ANFIS, a neuro-fuzzy hybridization technique that is used to predict the Mission_Success_Factor(), considering the weather conditions along the mission route.

Surface aviation weather observations include weather elements and forecasts pertaining to flying. A network of airport stations provides routine up-to-date surface weather information. Upper-air weather data is received from sounding balloons (radiosonde observations) and pilot weather reports that furnish temperature, humidity, pressure, and wind data. Aircraft in flight also report turbulence, icing and height of cloud tops. The weather radar provides detailed information about precipitation, winds, and weather systems. Doppler technology allows the radar to provide measurements of winds through a large vertical depth of the atmosphere. Terminal Doppler weather radars are used to alert and warn airport controllers of approaching wind shear, gust fronts, and heavy precipitation which could cause hazardous conditions for take-off, landing and diversion. Low-level wind shear alert systems provide pilots and controllers with information on hazardous surface wind conditions (on and near airbases) that create unsafe operational conditions. Visible, infrared and other types of images of clouds are taken from weather satellites in orbit. Weather is a continuous, multi-dimensional, spatio-temporally data intensive, dynamic and partly chaotic process. Traditionally, two main approaches for weather forecasting are followed: Numerical Weather Prediction and Analogue forecasting. For the AWGSS application, it is needed to consider the past weather conditions at given places of operation and predict the weather for simulation of mission tasks in real-time. In this paper, the ANFIS neuro-fuzzy hybridization technique is used to predict the weather conditions along the mission route and study the effects of weather in the virtual warfare scenario analysis in terms of pilot decisions in mission planning, performance of sensors, and target identification and damage assessment. 


\section{A Neuro-Fuzzy Hybridization Approach to Weather Prediction}

The weather agent has been designed using ANFIS to give the predicted Mission_Success_factor in weather constraints. In the following section, the neurofuzzy hybridization approach will be discussed. Both neural networks and fuzzy systems are dynamic, parallel processing systems that estimate input-output functions [6],[7],[8]. They estimate a function without any mathematical model and learn from experience with sample data. It has also been proven that 1) any rule-based fuzzy system may be approximated by a neural net and 2) any neural net (feed-forward, multilayered) may be approximated by a rule-based fuzzy system. Fuzzy systems can be broadly categorized into two families. The first includes linguistic models based on collections of IF-THEN rules, whose antecedents and consequents utilize fuzzy values. The Mamdani model falls in this group where the knowledge is represented as it is shown in the following expression.

$$
R^{i} \text { :If } X_{1} \text { is } A_{1}^{i} \text { and } X_{2} \text { is } A_{2}^{i} \ldots \ldots . \text { and } X_{n} \text { is } A_{m}^{i} \text {, then } y^{i} \text { is } B^{i}
$$

The second category, which is used to model the Weather prediction problem is the Sugeno-type and it uses a rule structure that has fuzzy antecedent and functional consequent parts. This can be viewed as the expansion of piece-wise linear partition represented as shown in the rule below.

$$
\begin{gathered}
R^{i}: \text { If } X_{1} \text { is } A_{1}^{i} \text { and } X_{2} \text { is } A_{2}^{i} \ldots \ldots \text { and } X_{n} \text { is } A_{m}^{i} \text {, then } y^{i}=a_{0}^{1}+a_{1}^{i} X_{1}+\ldots .+a_{n}^{i} X_{n} \\
\tilde{A} \cap \tilde{B}=\left\{\left(x, \mu_{\tilde{\mathrm{A}} \cap \tilde{\mathrm{B}}}(x)\right) \mid \mu_{\tilde{\mathrm{A}} \cap \tilde{\mathrm{B}}}(x)=\mu_{\tilde{\mathrm{A}}}(x)^{\wedge} \mu_{\tilde{\mathrm{B}}}(x)=\min \left(\mu_{\tilde{\mathrm{A}}}(x), \mu_{\mathrm{B}}(x)\right)\right\}
\end{gathered}
$$

The conjunction "and" Operation between fuzzy sets known as Linguistics, for the implementation of the Mamdani rules is done by employing special Fuzzy Operators called T-Norms [6]. The ANFIS uses by default the Minimum T-Norm which is the case here and it can be seen in the above equation 1. The approach approximates a nonlinear system with a combination of several linear systems, by decomposing the whole input space into several partial fuzzy spaces and representing each output space with a linear equation. Such models are capable of representing both qualitative and quantitative information and allow relatively easier application of powerful learning techniques for their identification from data. They are capable of approximating any continuous real-valued function on a compact set to any degree of accuracy [9],[10]. This type of knowledge representation does not allow the output variables to be described in linguistic terms and the parameter optimization is carried out iteratively using a nonlinear optimization method.

Fuzzy systems exhibit both symbolic and numeric features. Neuro-fuzzy computing [8],[11] is a judicious integration of the merits of neural and fuzzy approaches, enables one to build more intelligent decision-making systems. Neurofuzzy hybridization is done broadly in two ways: a neural network equipped with the capability of handling fuzzy information [termed fuzzy-neural network] and a fuzzy system augmented by neural networks to enhance some of its characteristics like flexibility, speed, and adaptability [termed neural-fuzzy system]. ANFIS is an adaptive network that is functionally equivalent to a fuzzy inference system and 
referred to in literature as "adaptive network based fuzzy inference system" or "adaptive neuro-fuzzy inference system" (Fig.3) [9],[10],[13],[14]. In the ANFIS model, crisp input series are converted to fuzzy inputs by developing triangular, trapezoidal and sigmoid membership functions for each input series. These fuzzy inputs are processed through a network of transfer functions at the nodes of different layers of the network to obtain fuzzy outputs with linear membership functions that are combined to obtain a single crisp output the predicted Mission_Success_Factor, as the ANFIS method permits only one output in the model. The following equations 2,3,4 correspond to triangular, trapezoidal and sigmoid membership functions.

$$
\mu_{s}(X)=\left\{\begin{array}{l}
0 \text { if } X<a \\
(X-a) /(c-a) \text { if } X \in[a, c) \\
(b-X) /(b-c) \text { if } X \in[c, b] \\
0 \text { if } X>b
\end{array} \quad(2) \quad \mu_{s}(X)= \begin{cases}0, & \text { if } X \leq a \\
(X-a) /(m-a), & \text { if } X \in(a, m) \quad(3) \\
1, & \text { if } X \in[m, n] \\
(b-X) /(b-n), & \text { if } X \in(n, b) \\
0, & \text { if } X \geq b\end{cases}\right.
$$

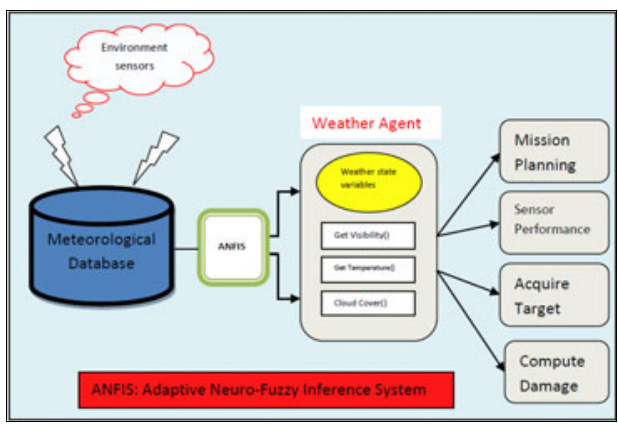

Fig. 3. Weather agent's architecture and behaviors

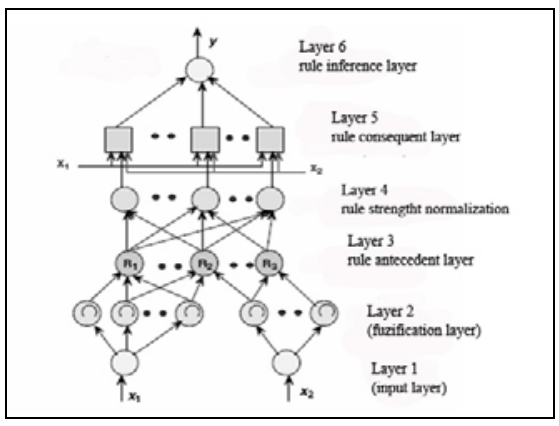

Fig. 4. ANFIS architecture to design the Weather agent

Weather conditions of interest to AWGSS [15],[16],[17],[18] are classified as Precipitation (Drizzle, Rain, Snow, Snow-grains, Ice crystals, Ice pellets and Hail), Obscuration (Mist, Fog, Dust, Sand, Haze, Spray, Volcanic ash, Smoke) and Others (Dust/Sand whirls, Squalls, Funnel cloud, Tornado or Water spout, Sandstorm, Duststorm). Temperature, Clouds, Height of cloud base, Wind speed and direction, Icing, Precipitation, Visibility, Fog, Mist, Rain, Thunderstorm, Haze, dust/sand whirls and squall speeds are quantified using linguistic fuzzy variables. Target Identification factor: Rapid and certain target detection and identification are the dominant factors in the success of all air-to-ground attacks. The ability of tactical fighters to penetrate enemy defenses and to acquire and identify ground targets successfully within weather constraints is a keystone of success in a mission. It has been observed that aerial observers respond to targets in a manner indicating that detection / identification represents a continuum rather than discrete phenomena. At one extreme the response is based on the ability to merely discriminate the existence of a military object among non-military objects (detection) [19],[20],[21]. At the other extreme the 
observer can describe the object in precise detail (identification). Factors considered for computing the Target Identification factor are target size, percent contrast, illumination, terrain, weather conditions, altitude and speed of the aircraft at time of target acquisition. Target Size: As target size increases, probability of correct target identification increases. It may vary from small to large tactical targets, including personnel, trucks, and tanks to big targets as bridges, runways and taxi-tracks. Contrast: Target/Ground Brightness Contrast is expressed as a percentage. Illumination: Detection performance increases as illumination increases. Effects of decreases in illumination occurring after sunset and before sunrise are very important and need to be considered. Terrain: Types of terrain have been defined in terms such as number of slope changes per unit area and average slope change. Four different terrain types have been defined-fairly smooth, moderately rough, rough, and very rough. As the roughness of terrain increases, percent terrain view decreases, and decrease in detection performance is observed. Weather: Temperature, humidity, and wind effects the performance of sensors (such as Radars) deployed, where as conditions such as Precipitation, icing, wind, visibility, fog, rain, date and time of operation, clouds, and storm effect the pilots' decisions in planning and executing the missions. Altitude: The relationship between altitude and target detection/identification is normally one in which there is assumed to be an optimal altitude; above and below this optimum altitude, detection is reduced. As altitude increases, detection performance decreases. As altitude is increased beyond an optimal point, detection probability falls off rapidly.

Data on all these factors are collected from meteorological department databases, handbooks and experimental field trials and heuristic knowledge from experts and defense analysts (in questionnaire form) are collected and recorded. They are then represented as decision matrices and decision trees which form the basis to design the membership functions and rules. The rules are then executed in the mission processing module and defuzzified to obtain the damage to target [22]. These results are then compared to the expected output and fine-tuned before storing in the rule base. A decision to include the new rule or not is provided to the commander. Missions and results of the missions are stored as a case-base for retrieval and reuse of missions plans in new situations. The following fuzzy linguistic variables used in the design of the game rules are as follows:

Mission_Success_Factor (with weather constraints): [1-10] \{Very Low: [0.0 - 3.5]; Low with Moderate Risk [2.5 - 5.5]; Medium with Controllable Risk [4.5 - 7.5]; High with Moderate Risk [6.5 - 8.0]; Very High with Low Risk [7.5-10.0]\} Temperature: [Very Low, Low, Moderate, High, Very High] Fog-Haze: [Shallow, Patches, Low Drifting, Blowing, Showers, Thunderstorm, Freezing, Partial] Wind-Speed: [Light, Moderate, Heavy] Clouds/Cloud Base: [Shallow, Patches, Low Drifting, Blowing, Showers, Thunderstorm, Partial]; [Height (ft)] Visibility: [Low, Medium, Clear]

Turbulence: [Clear, Low, Medium, Heavy] Storm/Squalls: [Clear, Low, Medium, Heavy] Sky Cover: [Clear, Few, Scattered, Broken, Overcast, Vertical Visibility]

Terrain: [1-100] \{Fairly Smooth [0 -22]; Moderately Rough [14 - 49]; Rough [45-81]; Very Rough [75 - 100]\}. Target Size (in feet): \{Very small: [0 -100]; Small: 
[70 - 190]; Medium sized: [160 - 300]; Large: [270 - 400]; Fairly Large: [360 500]; Extremely Large: [450 - 900]\} Damage: Offset (in meters): \{Very Less:[0-23]; Less:[16-36]; Medium: [34-57]; Large: [56-80]; Very Large [78-100]\} Weapon Target Match: [0 -10] \{Poor: [0-3.6]; Average: [3.36 - 6.669]; Good: [6.73 - 14.2]\}

Target Identification Factor: [0 -10] \{Very poorly identified: [0-1.19]; Poorly identified [0.96 - 2.43]; Average identification [2.34 - 5.61]; Good identification [5.43 - 7.55]; Excellent identification [7.35 - 10]\} Relative Damage (Damage relative to intended damage): $[0$ - 100] \{Mild: [0-18]; Moderate: [16-36]; Average: [34-57]; Severe: [56-80]; Fully Damaged: [78-96]\}.

Data from meteorological database is used to train the network to apply a hybrid method whose membership functions and parameters keep changing until the weather forecast error is minimized (Fig.5(a),(b)). Then the resulting model is applied to the test data of the mission time and places en-route from take-off base, target and landing base.

\section{Results Discussion}

The fuzzy variables are used to calculate the Mission success factor based on the prevailing weather conditions generated by the ANFIS model, target identification factor and firing of the rules to compute the relative damage to the target (Fig. 2). Offset is calculated using actual altitude, actual vertical flight path angle, actual wind speed and observed altitude, observed altitude, observed vertical flight path angle, observed wind speed by the weapon system trajectory calculation module and the aircraft speed as the input variables (Table 3). Offset is a measure of induced error, wind induced error, and vertical flight path angle induced error.

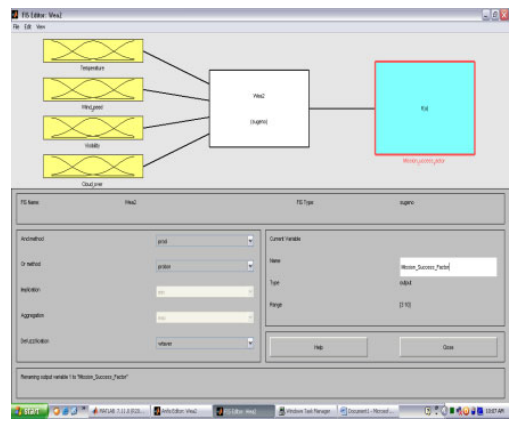

(a)

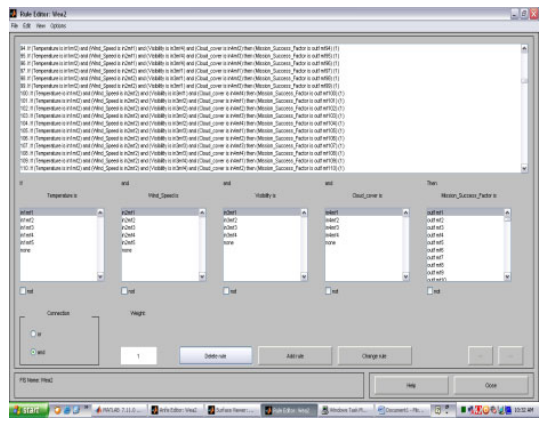

(b)

Fig. 4. (a),(b): ANFIS and Rules for computing Mission Success Factor

Case Mission ID \# 001: Consider a large area-target of size of $550 \mathrm{ft}$ to be attacked, where the fuzzy variables target-ground contrast $80 \%$, the terrain, rated 8 , is fairly smooth, aircraft altitude is $900 \mathrm{ft}$, aircraft range is $5000 \mathrm{ft}$ is flying at 100 knots speed. 
The target identification factor for this target is computed as "good" with value 7.32. (In the tables below * denotes the Missions planned and executed when considering the Weather conditions.)

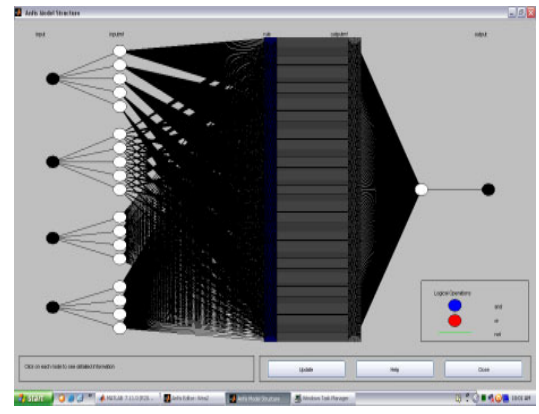

(a)

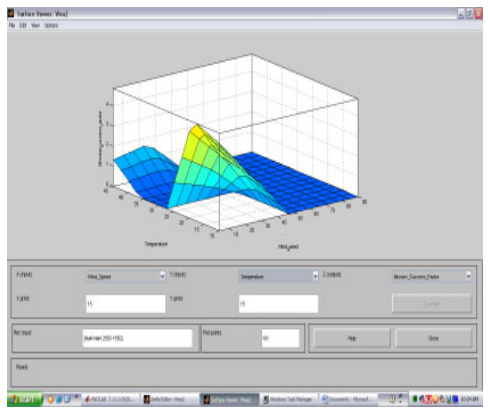

(b)

Fig. 5. (a),(b) ANFIS Rules and Surface Plot for the different Weather conditions in AWGSS

Table 1. Fuzzy Rules to determine the Mission Success factor in Weather conditions

\begin{tabular}{|l|l|l|l|l|l|l|l|l|}
\hline MissionID & Temperature & Fog-Haze & $\begin{array}{l}\text { Wind Speed } \\
(\mathrm{m} / \mathrm{s})\end{array}$ & $\begin{array}{l}\text { Clouds/ } \\
\text { Base }\end{array}$ & Visibility & Turbulence & $\begin{array}{l}\text { Storm/ } \\
\text { Squalls }\end{array}$ & $\begin{array}{l}\text { Mission } \\
\text { Success } \\
\text { Factor }\end{array}$ \\
\hline$\# 001$ & Moderate & Clear & Low-Drifting & Clear & Clear & Low & Clear & 8.4 \\
\hline$\# 001 *$ & Very Low & Moderate & High & Low & Poor & High & Clear & 3.7 \\
\hline$\# 002$ & Moderate & Clear & Moderate & Scattered & Clear & Low & Clear & 9.8 \\
\hline$\# 002 *$ & Very High & Haze & High & Low & Poor & Low & Squall & 7.1 \\
\hline
\end{tabular}

Table 2. Fuzzy Rules to determine the Target Identification factor

\begin{tabular}{|l|l|l|l|l|l|l|l|l|l|}
\hline MissionID & $\begin{array}{l}\text { Target } \\
\text { Size(ft) }\end{array}$ & $\begin{array}{l}\text { Target- } \\
\text { Ground } \\
\text { Contrast\% }\end{array}$ & $\begin{array}{l}\text { Illumination } \\
\text { (foot candles) }\end{array}$ & Terrain & $\begin{array}{l}\text { Weather_ } \\
\text { Mission } \\
\text { Success } \\
\text { Factor }\end{array}$ & $\begin{array}{l}\text { Aircraft } \\
\text { Altitude } \\
\text { (feet) }\end{array}$ & $\begin{array}{l}\text { Aircraft } \\
\text { Range } \\
\text { feet) }\end{array}$ & $\begin{array}{l}\text { Aircraft } \\
\text { Speed } \\
\text { (knots) }\end{array}$ & $\begin{array}{l}\text { Target } \\
\text { Identification } \\
\text { Factor }\end{array}$ \\
\hline$\# 001$ & 550 & 80 & 40 & 8 & 8.4 & 900 & 5000 & 100 & 7.32 \\
\hline$\# 001^{*}$ & 550 & 45 & 20 & 8 & 3.7 & 900 & 5000 & 80 & 5.67 \\
\hline$\# 002$ & 550 & 80 & 60 & 7 & 9.8 & 750 & 4000 & 80 & 8.03 \\
\hline$\# 002^{*}$ & 550 & 45 & 30 & 7 & 7.1 & 750 & 4000 & 60 & 6.43 \\
\hline
\end{tabular}

Table 3. Fuzzy Rules to compute the Relative damage to target

\begin{tabular}{|l|l|l|l|l|l|l|}
\hline MissionID & $\begin{array}{c}\text { Offset } \\
(\text { meters })\end{array}$ & $\begin{array}{c}\text { Target Radius } \\
(\mathrm{km})\end{array}$ & $\begin{array}{c}\text { Weapon-Target } \\
\text { Match }\end{array}$ & $\begin{array}{c}\text { Weapon } \\
\text { Delivery Mode }\end{array}$ & $\begin{array}{c}\text { Target } \\
\text { Identification } \\
\text { Factor }\end{array}$ & $\begin{array}{l}\text { Relative } \\
\text { damage }\end{array}$ \\
\hline$\# 001$ & 29.03 & 0.09 & 6 & 6 & 7.32 & 28.91 \\
\hline$\# 001^{*}$ & 37.54 & 0.09 & 6 & 6 & 5.67 & 13.55 \\
\hline$\# 002$ & 6.07 & 90.0 & 9 & 9 & 8.03 & 88.74 \\
\hline$\# 002^{*}$ & 12.65 & 90.0 & 9 & 9 & 6.43 & 65.92 \\
\hline
\end{tabular}


Table 4. Fuzzy attributes to determine the offset of the weapon from the intended target

\begin{tabular}{|l|l|l|l|l|l|l|l|l|}
\hline $\begin{array}{c}\text { Mission } \\
\text { ID \# }\end{array}$ & $\begin{array}{c}\text { Apparent } \\
\text { Altitude(km) }\end{array}$ & $\begin{array}{l}\text { Apparent } \\
\text { Angle } \\
\text { (degrees) }\end{array}$ & $\begin{array}{l}\text { Apparent } \\
\text { Wind } \\
\text { velocity } \\
(\mathrm{km} / \mathrm{hr})\end{array}$ & $\begin{array}{l}\text { Actual } \\
\text { Altitude(km) }\end{array}$ & $\begin{array}{l}\text { Actual } \\
\text { Angle(degrees) }\end{array}$ & $\begin{array}{l}\text { Actual } \\
\text { Wind } \\
\text { velocity } \\
(\mathrm{km} / \mathrm{hr})\end{array}$ & $\begin{array}{l}\text { Aircraft } \\
\text { speed(km/hr) }\end{array}$ & $\begin{array}{l}\text { Offset } \\
(\mathrm{meters})\end{array}$ \\
\hline 001 & 1.65 & -26.9 & -25.24 & 1.67 & -26.9 & -25.24 & 829.8 & 29.03 \\
\hline $002^{*}$ & 1.65 & -26.9 & -23.9 & 1.67 & -26.9 & -25.24 & 820.1 & 37.54 \\
\hline 002 & 1.65 & -25.2 & -28 & 1.65 & -25.2 & -30.4 & 830.2 & 6.07 \\
\hline $002^{*}$ & 1.65 & -25.2 & -22 & 1.65 & -25.2 & -30.4 & 824.7 & 12.65 \\
\hline
\end{tabular}

In this mission, on firing the rules for inference, the offset from the desired point of impact is $29 \mathrm{~m}$, considered "less"(i.e. fairly accurate targeting); weapon-target match is 6 (average), "good" target identification factor 7.32, the relative damage caused is 28.92 which is a "moderate" damage to the target. We consider two scenarios of weather conditions at the given place and time or the mission plan (Fig.2). Weather conditions are identified based on the place and time of missions. The ANFIS model computes the Mission_Success_Factor as 8.4 when no weather conditions are considered, and reduces to 3.7 when weather conditions are considered in the AWGSS (Table1, 2). These conditions also reduce the Relative Damage from 28.91 to 13.55 (Table 3) and offset of the weapon hitting away from the intended target increased from 29.03 to 37.54 (Table 4).

Case Mission ID \# 002: Another mission planned by the commander where a similar target is chosen with the fuzzy variables as shown in Tables 1 and 2 . While the offset has reduced to $6 \mathrm{~m}$, considered "very less" (i.e. very accurate targeting), choosing a different weapon system and delivery improved the weapon-target match to 9 ("good"), and mode of weapon delivery 9, the target identification factor also improved to 8.033 (considered "excellent"), and the relative damage caused is 88.74 , which is a "substantial" damage to the target (Tables 2, 3). Weather conditions are again identified based on the place and time of missions. The ANFIS gives the Mission_Success_factor as 9.8 when no weather conditions are considered, and reduces to 7.1 when weather conditions are considered in the AWGSS (Table1, 2). These conditions also reduce the Relative Damage from 28.91 to 13.55 (Table 3) and offset of the weapon hitting away from the intended target increased from 6.07 to 12.05 (Table 4). These attributes form the antecedents of the fuzzy rule and the consequent is shown in the last column of the tables. For all the missions that the pilots plan in the wargame exercises, these fuzzy game rules are used to infer the expected damage caused to the target. These missions form a part of a case-base which is used as part of the 'learning' by the system for future instructional use.

\section{Conclusions}

A novel approach using ANFIS is presented in this research paper. More specifically, a neuro-fuzzy hybridization technique is employed to model the operations of the weather agent in a virtual warfare analysis system called AWGSS that is designed using an agent-based architecture. The system is applied to compare the results obtained in the presence of weather in combat simulation exercises. The results that 
are predicted by the weather agents after a pilot application and the rules that are generated to predict the Mission_Success_Factor are found to be very satisfactory in predicting the mission's performance in the presence of different weather conditions over different regions of the mission route. This concept induces a realistic methodology to introduce weather conditions in the AWGSS by using ANFIS as the reasoning and inference system in the weather agent. A future research effort will include working on ways for optimizing the rules for specific weather conditions like extreme weather phenomena and also on the improvement of the overall system's performance. Of course the whole idea and the framework of the developed system can be applied also in other wider problems like watching and taking real time measures for the cases of natural disasters.

Acknowledgments. We thank Air Marshal "Doc" Vaidya and Air Cmde S P Ojha, College of Air Warfare for the useful insights and discussions on air warfare tactics and game rules development; and the scientists of the air wargaming team for their enthusiasm.

\section{References}

1. Vijay Rao, D.: The Design of Air Warfare Simulation System. Technical report, Institute for Systems Studies and Analyses (2011)

2. Vijay Rao, D., Jasleen, K.: A Fuzzy Rule-based approach to design game rules in a mission planning and evaluation system. In: Papadopoulos, H., Andreou, A.S. (eds.) 6th IFIP Conference on Artificial Intelligence Applications and Innovations, Cyprus. Springer, Heidelberg (October 2010)

3. Vijay Rao, D., Saha, B.: An Agent oriented Approach to Developing Intelligent Training Simulators. In: SISO Euro-SIW Conference, Ontario, Canada (June 2010)

4. Banks, J. (ed.): Handbook of Simulation: Principles, Methodology, Advances, Applications, and Practice. John Wiley and Sons, New York (1998)

5. Maqsood, I., Khan, M.R., Abraham, A.: An ensemble of neural networks for weather forecasting. Nerual Computing \& Applications 13, 112-122 (2004)

6. Cox, E.: The Fuzzy Systems Handbook, 2nd edn. Academic Press, New York (1999)

7. Castellanos, F., James N.: Average Hourly Wind speed forecasting with ANFIS. In: 11th American Conference on Wind Engineering, San Juan, Puerto Rico (June 2009)

8. Taher, J., Zomaya, A.Y.: Artificial Neural Networks in Handbook of Nature-Inspired and Innovative Computing. In: Zomaya, A.Y. (ed.) Integrating Classical Models with Emerging Technologies, pp. 147-186. Springer, USA (2006)

9. Jang, J.S.R.: ANFIS: Adaptive Network-based fuzzy inference systems. IEEE Trans. on Systems, Man and Cybernetics 23(3), 665-685 (1993)

10. Jang, J.S.R., Sun, C.T., Mizutani, E.: Neuro-Fuzzy and Soft Computing: A Computational approach to Learning and Machine Intelligence. Prentice Hall, New Jersey (1997)

11. Pedrycz, W.: Computational Intelligence-An Introduction. CRC Press, USA (1998)

12. Hiramatsu, A., Van-Nam, H., Yoshiteru, N.: A Behavioral decision model based on Fuzzy Targets in decision making using weather information. Journal of Advanced Computational Intelligence and Intelligent Informatics 12(5), 435-442 (2008)

13. Mitra, S., Hayashi, Y.: Neuro-Fuzzy Rule Generation: Survey in Soft Computing Framework. IEEE Trans. Neural Networks 11(3), 748-768 (2000) 
14. Mendel, J.M.: Uncertain Rule-Based Fuzzy Logic Systems-Introduction and New Directions. Prentice Hall PTR, Upper Saddle River (2001)

15. Federal Aviation Administration, The Weather Forecast, R \& D Review (1), USA (2006)

16. Pierce, C., Hirsch, T., Bennett, A.C.: Formulation and evaluation of a post-processing algorithm for generating seamless, high resolution ensemble precipitation forecasts, Forecasting R \& D Tech. Report No. 550, Exeter, UK (November 2010)

17. Bovis, K., Dow, G., Dumelov, R., Keil, M.: An assessment of deployment strategies for targeting observations, Tech. Rep. No. 515, Meteorological research and Development, UK (2008)

18. Das, A.K., Saxena, R., Roy Bhowmik, S.K.: NWP Inputs of IMD for use in Operational Fog Forecasting, IMD Forecaster-Users interactive workshop Fog monitoring and Forecasting services, India Meteorological Services, New Delhi (December 2010)

19. Iliadis, L.: Intelligent Information Systems and Risk estimation. A Stamoulis publ. (2007)

20. Meitzler, T.J., Singh, H., Sohn, E.: Fuzzy Logic Technique to Determine Search Time and Probability of Detection for Targets of Interest in Background Scenes, TACOM Research Development and Engineering Center, Warren, MI (2002)

21. Celminss, A.: Vulnerability assessment of fuzzy targets. Fuzzy Sets and Systems 68(1), 2938 (1994)

22. MATLAB Fuzzy Logic Toolbox 